\title{
Study on the Application of Carbohydrate Derivatives in Food Industry
}

\author{
Xiuchuan $\mathrm{Hu}^{1, \mathrm{a}}$
}

\author{
${ }^{1}$ Wuhan Bioengineering Institute of Food Science and Engineering, Wuhan, Hubei, 430415 \\ a email
}

Keywords: Carbohydrate Derivatives, Food Industry, Application

\begin{abstract}
Carbohydrate is a kind of natural macromolecule compound, which is linked by aldose or ketose through the glycosidic linkages of polymers and is present in higher plants, algae, fungi and animals. It is the most abundant natural content Biopolymers. According to its sources, polysaccharides can be divided into animal polysaccharides, plant polysaccharides, microbial polysaccharides and artificial biosynthesis of polysaccharides. It has a variety of biological functions, constitute animals and plants and microbial extracellular structural substances (such as cellulose and chitin) and energy substances (such as starch and glycogen). This paper studies physicochemical properties of polysaccharides, carbohydrate and its derivatives and their applications in the food industry.
\end{abstract}

\section{Introduction}

Carbohydrate is the biological macromolecules composed of $\mathrm{C}, \mathrm{H}, \mathrm{O}$ and it can be divided into monosaccharides, disaccharides and polysaccharides. Carbohydrates are widely distributed in the nature of a class of important organic compounds. Daily consumption of sucrose, starch in grain, plant cellulose in the body, glucose and other blood in the human body are carbohydrates. Carbohydrate plays an important role in the process of life activities, and is the main source of all the energy needed to sustain life activities. The most important sugar in plants is starch and cellulose, the most important polysaccharide in animal cells is glycogen.

Chitosan is the only basic polysaccharide in natural polysaccharides, and it is also one of the few natural products with chargeability. It has good film-forming, hygroscopicity, permeability, permeability and biodegradability. Its molecular structure contains many hydroxyl and amino, and therefore easy to chemical modification and modification. Chitosan modification mainly utilizes the reactivity of various functional groups in the molecule to produce specific chemical reactions, including graft copolymerization, methylolation, acylation, etherification, N-alkylation, Esterification reaction, crosslinking reaction, oxidation - reduction reaction and complexation reaction to produce a series of different properties of chitosan derivatives, they can be biodegradable and it is a non - toxic, non - polluting polymer materials .

Polysaccharide is a kind of natural macromolecule compound. It is a kind of polymer which is linked by aldose or ketose through glycosidic bond. It exists in higher plants, algae, fungi and animals. It is the most abundant biological substance in nature polymer. According to their sources, polysaccharides can be divided into animal polysaccharides, plant polysaccharides, microbial polysaccharides and artificial biosynthesis of polysaccharides, with a variety of biological functions, constitute animals and plants and microbial extracellular structural substances (such as cellulose and chitin) Energy substances (such as starch and glycogen); at the same time, as the core of life processes play a role in the molecules such as genetic material, enzymes, antibodies, hormones, membrane proteins and lipids, polysaccharides are an indispensable part of the polysaccharide can Protein, lipid-forming glycoprotein, lipopolysaccharide, in the cell recognition, secretion and protein processing and transfer plays an important role; polysaccharide also has a wide range of pharmacological effects, such as strengthening the body immunity, Anti-tumor, anti-virus, hypoglycemic, protection of gastrointestinal function and other activities, health care products and pharmaceutical research and development of hot spots, and because of polysaccharide has a special physical and chemical properties, widely used in the food industry. 


\section{Animal Polysaccharide Application}

When the concentration of chitosan solution was $0.4 \%$, it had strong effects on Gram-negative bacteria, Escherichia coli, Escherichia coli, Fluorescent pseudomonas, Proteus vulgaris, Staphylococcus aureus, Bacillus subtilis and some yeasts and molds Antibacterial. If the chitosan dissolves in the acidic aqueous solution, the long-chain partial hydrolysis will occur and it was known as the degradation of $0.1 \%$ chitosan acetic acid solution can completely inhibit Staphylococcus aureus, Pseudomonas aeruginosa and the production of Candida tropicalis. In the seasoning added in the brewing vinegar in chitosan it can inhibit bacteria 7 days, with the use of sodium acetate can be increased to 12 days, the better.

As chitosan has significant bacteriostasis and natural film-forming properties, it has been used as a fresh-keeping material for fruits and vegetables, such as chitin and chitosan as the main raw materials, and sprayed on the surface of fruit and vegetable. Layer can keep the fruit intact for more than 9 months. However, due to chitin, chitosan water insoluble, as the protective layer of fruits and vegetables is difficult to wash off, so now more water-soluble chitosan of derivatives, such as carboxymethyl chitosan sprayed on fruits and vegetables, the membrane also has a selective permeability of gas, can inhibit respiration, and have better preservation of fruits and vegetables, while easy to clean. Japan will chitosan and Calcium lactate or calcium acetate heat treatment into a highly effective bactericidal activity, safe and non-toxic water-soluble preservative, American scholars with N-carboxymethyl chitosan made meat preservative, can be used for cooked beef, fish, Poultry, eggs and daily food preservative preservation, while oral bacteria have an exceptionally strong antibacterial capacity.

The use of chitosan and organic acids to produce salt and chitosan as a flocculant used in the industry has its unique advantages. Current industrial application of cationic industrial flocculant is the vast majority of synthetic polymers. Very toxic and it is not suitable for food industry application. The chitosan can be used in food industry because of its non-toxic side effect, biodegradation and secondary pollution. It can effectively remove the suspended matter and most phenol acidity makes the juice clear and transparent, without affecting one of the nutrients and flavor. With rice fermentation produced wine, the general turbidity of 350b, and per liter of wine by adding $3 \mathrm{ml}$ of $0.2 \%$ chitosan tartaric acid solution and $1 \mathrm{ml} 0.1 \%$ sodium polyacrylate aqueous solution, stirring and standing, the suspension immediately precipitated by filtration can be turbidity of $5 \mathrm{~b}$ of the sake of the original sugar syrup contains a variety of organic colloidal substances, cellulose, lime and other small suspended , Sugar must be the first to make the separation of chitosan is the ideal flocculant syrup, sugar juice added $2 \sim 50 \mathrm{ppm}$ chitosan can quickly make suspended solids in the juice agglutination, the formation of aggregates particles Large, rapid settlement, easy to filter. In the food industry wastewater treatment has a large number of applications, the author has been published.

Chitosan film can be made into food packaging materials, such as packaging film, sausage clothing, etc., it has antibacterial effect, longer than other film preservation, and mixed with chitosan and starch film, alkali treatment after insoluble in cold water, do not dissolve in hot water, tensile strength, edible, oil, can also be packaged liquid.

\section{Plant Polysaccharides}

Plant polysaccharides is a plant in the life activities of the formation of polymer or complex, such as intracellular storage of starch and polysaccharides and other non-starch storage polysaccharides, constitute the cell pectin substances, cellulose and semi Cellulose, higher plants of the roots, skin, leaves, seeds and flowers contained in the gum and viscose are also polysaccharide protein complexes. Plant polysaccharides has many biological activities, such as immune regulation, anti-tumor, hypoglycemic, anti-radiation, anti-bacterial anti-virus, protecting the liver and other health effects, is one of the most studied polysaccharide, Polysaccharides extracted from the plant and widely used in medicine and health food research and development. As the plant polysaccharide has the advantages of renewable, less investment, low cost, little pollution, high utilization rate, it 
has become a hotspot in food industry research and is widely used in food processing and production.

Cellulose is the main component of the plant cell wall, is glucose molecules through the $\beta$ - $(1,4)$ - glycosidic linkages formed by glucan. Cellulose is the most widely distributed and most abundant polysaccharide in nature. It is often used as a food packaging material in the food industry. It is biodegradable, absorbable, edible, safe and anti-oxidant and has good CO2 and O2 It can be used as an active packaging material for fruits, vegetables, legumes and meat products for moisturizing, anti-wetting and anti-oxidation.

The packaging material developed with cellulose as material has fruit paper and seaweed paper. The Institute has successfully extracted dietary fiber from okara and processed it into edible paper. In recent years, at home and abroad on the modified cellulose edible packaging film also has extensive and in-depth study, sodium alginate and sodium carboxymethyl cellulose film can be made of good food packaging film. HPMC is a derivative of cellulose contained in wood pulp. In the food industry, HPMC is usually used as a thickener, emulsifier, stabilizer and water retention agent for ice cream, bread, and salad dressing. . The United States generally used microcrystalline cellulose manufacture of frozen food and cold drinks and cooking sauces and cooking; East Germany began in 1975 to produce microcrystalline cellulose, and the use of microcrystalline cellulose and its products as additives manufacturing salad oil, Dextrin condiment.

Dietary fiber is a kind of not easily digested food nutrients, mainly from the plant cell wall, by the hemi-cellulose, cellulose, lignin and pectin, gum, mucus and other components, is the food has a certain physiological function of chemical composition. Dietary fiber in the past is considered to be "waste", and now people have recognized that it protects human health, prolong life has an important role. It is known that proteins, fats, carbohydrates, minerals, vitamins and water are the six essential nutrients the body needs, and dietary fiber is called the seventh nutrient. Moderate consumption of dietary fiber can improve the cavity and dental function, promote the growth of beneficial intestinal flora, prevention and treatment of constipation, help weight loss, but also can improve the symptoms of diabetes.

Soybean polysaccharide is mainly soybean protein processing or bean curd and other soy products processing by-products bean dregs fiber as the main raw material, by pretreatment, enzymolysis, separation, decolorization, sterilization, drying process refined. Soybean polysaccharide is composed of galactose, arabinose, galacturonic acid and glucose and other components, the composition of the structure is poly-galacturonic acid and the main chain with galactan and arabinose side chain similar to the spherical structure. Soybean polysaccharide in addition to the characteristics of general dietary fiber, but also has the stability of emulsification and emulsification, acidic conditions on the stability of protein particles, anti-adhesion, film and foam stability and other characteristics of the food industry have more application.

Soybean polysaccharide is a glycoprotein, the protein part can quickly adsorbed to the surface of the oil droplets, sugar chain part of the water phase into the water droplets around the formation of a thick layer of hydration film, the role of steric hindrance by the oil droplets stability, The stability of the emulsion is not affected by the $\mathrm{pH}$ value of the system, the salt ion concentration, and has a certain thermal stability. Soybean polysaccharide as emulsifier has been widely used in coffee cream, emulsified seasoning, coffee mate and milk yolk cream, the traditional formula often add sodium carboxymethyl cellulose, carrageenan, xanthan gum, sodium alginate, melon Such as margarine, salad dressing oil, cream sauces and other food products, such as soy sauce, soy sauce, etc., as a thickening of the product thickening, taste sticky, with soybean polysaccharide as stabilizer milk drinks taste is more refreshing; Soybean polysaccharide can be partially replaced by fat, to maintain moisture, increase product sense of adhesion and adhesion.

Soybean polysaccharide can be used in rice, fried and non-fried instant noodles, noodles, noodles, rice noodles, pasta, bread, Cantonese rice cakes, Cantonese-style rice noodles, Yunnan noodle, etc., can form rice and flour surface hydration layer It can prevent the sticking of rice and flour noodles, so that the food is more refreshing and delicious, such as Japanese sushi, non-fried instant noodles, Japanese rice noodles, Japanese convenience rice and fresh wet surface. 


\section{Conclusion}

Carbohydrates and their derivatives have been widely used in the food industry due to their unique physicochemical properties and their wide biological activities. Their antibacterial properties are used for food preservation. Their good film-forming and biodegradability properties are used for food packaging materials. Their emulsifying and fluidity properties are used for food emulsifier thickener and so on. Carbohydrates and its derivatives have become an important raw material and additives in the food industry.

\section{References}

[1] Devies Jw, Prosky L, Li B. A historical perspective on defining dietary fiber[J]. Cereal Food World, 1999, 44 (5): 367-369.

[2] Anderson J W, Baird P. Health benefits of dietary fiber [J]. Nutrition Reviews, 2009, 67 (4): 188-205.

[3] Wang Siyuan, Qi Junru, Yang Xiaoquan. Effect of soybean polysaccharide on rice starch gelatinization and gel properties[J]. 2011, 30(7): 155-158.

[4] Luo Xuegang. Preliminary study on the application of high-strength edible konjac glucomannan film[J]. Natural Product Research and Development, 2000, 12 (4): 12-16.

[5] Guo Qiaoling, Xie Jianhua, Pang Jie. Study on the Effect of coating and cold shock treatment on the quality of Guanxi pomelo [J]. Preservation and Processing Technology, 2007, 7 (2): 17-19.

[6] Huang Jie, An Qiufeng. Advances in guar gum research [J]. Food Research and Development, 2011, 32 (1) 